\title{
Incidência do parto prematuro em gestantes com COVID-19: uma revisão integrativa
}

\author{
Incidence of preterm birth in pregnant women with COVID-19: an integrative review
}

Incidencia de parto prematuro en mujeres embarazadas con COVID-19: una revisión integradora

Veronica Arruda Barreto Souza

ORCID: https://orcid.org/0000-0001-7716-8803

Centro Universitário Aparício Carvalho, Brasil E-mail: vevearrudasouza@gmail.com

Antonio Rodrigues da Silva Neto ORCID: https://orcid.org/0000-0003-0054-510X

Universidade Estadual do Piauí, Brasil

E-mail: antonionettors5@gmail.com

Dakson Douglas Araújo

ORCID: https://orcid.org/0000-0003-0102-7240

Universidade Federal do Delta do Parnaíba, Brasil

E-mail: dacksondouglas@gmail.com

Ana Maria Romani

ORCID: https://orcid.org/0000-0001-8909-9331 Centro Universitário Aparício Carvalho, Brasil

E-mail: ana.romani07@gmail.com

Nayara Laís Viterbo

ORCID: https://orcid.org/0000-0001-8856-2865 Centro Universitário São Lucas, Brasil E-mail: nayaralaisviterbo@gmail.com

Naimi de Souza França Barroso

ORCID: https://orcid.org/0000-0001-8796-5507

Faculdades Integradas Aparício Carvalho, Brasil E-mail: naimibarroso@hotmail.com Amanda de Oliveira Botelho

ORCID: https://orcid.org/0000-0003-2665-1592 Centro Universitário São Lucas, Brasil

E-mail: amandabotelho.0490@ outlook.com

Letícia Fernanda de Magalhães

ORCID: https://orcid.org/0000-0002-0043-0049

Centro Universitário Aparício Carvalho, Brasil E-mail: lefermagalhaes41@ gmail.com Andhressa Tocashiki da Costa

ORCID: https://orcid.org/0000-0002-7500-6285

Universidade Federal de Rondônia, Brasil

E-mail: andhressatocashiki@gmail.com

Erica Ravena Alves Campelo

ORCID: https://orcid.org/0000-0002-3269-6458

Centro Universitário Santo Agostinho, Brasil E-mail: erica.r.campelo@hotmail.com

Márcia Maria Alves do Nascimento

ORCID: https://orcid.org/0000-0002-1470-5453 Universidade Estadual do Piauí, Brasil E-mail: m.m.nascimentto@gmail.com

Joseane Lima de Oliveira

ORCID: https://orcid.org/0000-0002-7560-6395

Centro Universitário UNINOVAFAPI, Brasil

E-mail: joseaneloliveira@ @otmail.com

Sofia Saldanha De Oliveira Marques

ORCID: https://orcid.org/0000-0003-0934-6051

Centro Universitário São Lucas, Brasil

E-mail: sofiasaldanha_om@hotmail.com

\section{Resumo}

A COVID-19 é uma doença que atingiu o mundo todo. Sendo uma patologia bastante estudada e com grande variação. Sendo uma infecção de grau de multiplicação e contaminação rápida e generalizada, obrigou a organização mundial da saúde a decretar estado de pandemia. Uma doença que pode levar a óbito rapidamente, principalmente, aqueles que possuem comorbidade, desta forma, começou se a ter um cuidado redobrado com as puérperas, pois 
devido as alterações fisiológicas da gravidez, essas tendem a evoluir para a síndrome respiratória aguda grave. Com isso, este estudo tem como objetivo analisar as complicações geradas pela síndrome respiratória aguda por SARSCoV-2 para a gestante e a associação entre a infecção e o parto prematuro. Que apresenta metodologia de revisão integrativa, de natureza exploratória e descritiva com abordagem qualitativa, que teve suas buscas nas plataformas de bases PubMed e ScinceDirect, de artigos de 2020 e 2021. A associação entre a infecção por SARS-coV-2 e o parto prematuro, ainda não está totalmente estabelecida, e alguns autores se divergem quanto opiniões e estudos. Dois pontos importantes foram discutidos que é a abordagem clínica mediante a realização do pré-natal em pacientes acometidas por COVID-19 e as complicações geradas pela síndrome respiratória aguda por SARS-CoV-2 para a gestante. Desta forma, é uma temática que apresenta lacunas e repostas ainda não consolidadas que carece de estudos que fomente essa holística.

Palavras-chave: COVID-19; Grávidas; Parto prematuro.

\begin{abstract}
COVID-19 is a worldwide disease. Being an unknown pathology and with great variation. As an infection with a rapid and widespread degree of multiplication and contamination, it forced a world health organization to declare a pandemic state. A disease that can quickly lead to death, especially those with comorbidity, thus began to take extra care with postpartum women, because due to the physiological changes of pregnancy, they tend to evolve into a severe acute respiratory syndrome. Thus, this study aims to analyze the complications generated by the acute respiratory syndrome caused by SARS-CoV-2 for pregnant women and the association between infection and preterm birth. Which presents an integrative review methodology, exploratory and descriptive in nature with a qualitative approach, which was searched in the PubMed and ScinceDirect database platforms, from articles from 2020 and 2021. The association between SARS-coV-2 infection and premature birth, is not yet fully praised, and some authors differ as to opinions and studies. Two important points were discussed, which is the clinical approach through prenatal care in patients affected by COVID-19 and as complications generated by the acute respiratory syndrome caused by SARS-CoV-2 for pregnant women. In this way, it is a theme that presents gaps and unconsolidated answers that lacks studies that encourage this holistic approach.
\end{abstract}

Keywords: COVID-19; Pregnant; Premature birth.

\begin{abstract}
Resumen
COVID-19 es una enfermedad mundial. Siendo una patología desconocida y con gran variación. Como una infección con un grado rápido y generalizado de multiplicación y contaminación, obligó a la organización mundial de la salud a declarar un estado pandémico. Una enfermedad que puede conducir rápidamente a la muerte, especialmente a aquellas con comorbilidad, por lo que se ha tenido un mayor cuidado con las mujeres posparto, ya que, debido a los cambios fisiológicos del embarazo, tienden a evolucionar hacia un síndrome respiratorio agudo severo. Así, este estudio tiene como objetivo analizar las complicaciones que genera el síndrome respiratorio agudo causado por el SARS-CoV-2 en mujeres embarazadas y la asociación entre infección y parto prematuro. El cual presenta una metodología de revisión integradora, de carácter exploratorio y descriptivo con enfoque cualitativo, la cual fue buscada en las plataformas de base de datos PubMed y ScinceDirect, a partir de artículos de 2020 y 2021. La asociación entre infección por SARScoV-2 y parto prematuro, no es aún plenamente establecido, y algunos autores difieren en cuanto a opiniones y estudios. Se discutieron dos puntos importantes, a saber, el abordaje clínico a través de la atención prenatal en pacientes afectadas por COVID-19 y las complicaciones generadas por el síndrome respiratorio agudo causado por SARS-CoV-2 en mujeres embarazadas. De esta manera, es un tema que presenta brechas y respuestas que aún no están consolidadas, que carece de estudios que fomenten este enfoque holístico.
\end{abstract}

Palabras clave: COVID-19; Embarazada; Nacimiento prematuro.

\title{
1. Introdução
}

Em dezembro de 2019, o primeiro caso de COVID-19 foi registrado na cidade de Wuhan, na China, tratava-se de uma nova cepa do coronavírus, capaz de infectar seres humanos e apresentou um alto grau de letalidade (Su et al., 2016; Sousa et al., 2020). Esse vírus apresenta-se com grande potencial de multiplicação, e também de contaminação, por conseguinte logo se espalhou por toda a Ásia, seguindo para a Europa, obrigando assim, em março de 2020, à Organização Mundial de Saúde (OMS) a decretar um estado de pandemia. Consequentemente, medidas para combater o patógeno foram criadas como isolamento social, uso de máscaras de proteção, fechamento do comércio e suspensão de atividades não essenciais, numa tentativa de combater o vírus (WHO, 2020; Silva et al., 2020).

A nova variante do coronavírus acomete principalmente o trato respiratório, comprometendo a fisiologia pulmonar e o estado de saúde do paciente. No entanto, a doença se manifesta de diferentes formas nos infectados, podendo ser uma simples 
infecção assintomática ou um preocupante quadro podendo levar ao óbito após complicações da doença, como a síndrome respiratória aguda grave (SARS) (Gorbalenya et al., 2020).

$\mathrm{O}$ vírus SARS-CoV apresenta alto risco morbidade, fator este que contribuiu também para o cuidado redobrado com a gestante. Alguns dos sintomas manifestados por esse grupo são febre, tosse seca, porém, em mulheres na segunda metade da gestação, há outros sintomas como por exemplo síndrome respiratória aguda grave (Zaigham \& Anderson, 2020). Existe também a manifestação de sintomas graves em gestações com o diagnóstico do COVID-19 revelando um quadro de aborto espontâneo; ruptura prematura de membranas; restrição de crescimento intrauterino; sofrimento fetal e trabalho de parto e parto prematuros (Mascarenhas et al., 2020; Da Costa et al., 2020).

As alterações fisiológicas da gravidez, como o aumento do consumo de oxigênio e imunidade celular alterada, tendem a ocasionar predisposição a infecções pulmonares com piores desfechos a gestantes, como o COVID-19, ocasionando a síndrome respiratória aguda (Pirjanir, et al., 2020). Dentre os riscos associados à gestante com diagnóstico positivo para COVID-19, é importante ressaltar o parto pré-termo/parto prematuro que ocorre ao menos três semanas antes da data prevista, as 40 semanas de gestação.

O parto prematuro ocorre antes das 37 semanas de gestação e após ultrapassar 20 ou 22 semanas de gestação (Bhering et al., 2021). Tendo em vista, os riscos associados à prematuridade, obtêm-se uma taxa de aproximadamente $47 \%$ de mortalidade e morbidade de recém-nascidos prematuros, provenientes de complicações por COVID-19 (Trapan júnior et al., 2020).

Uma vez que, estudos realizados por Liu et al. (2020) relataram que pacientes graves de COVID-19 foram submetidas a cesarianas de emergência por conta de vários fatores clínicos, sendo a maioria parto prematuro. Visto que, um estudo realizado pelo Centro de Controle de Doenças (CDC) dos Estados Unidos, indicou que mulheres grávidas com COVID-19 são mais propensas a desenvolver sintomas graves e as chances de sofrer um parto prematuro ou sofrerem perda gestacional são muito maiores. Além disso, estudos realizados por Allotey et al. (2020) evidenciaram taxas elevadas associadas ao risco de partos pré-termo em pacientes infectadas.

Portanto, o presente estudo tem como objetivo analisaras complicações geradas pela síndrome respiratória aguda por SARS-CoV-2 para a gestante e o recém-nascido prematuro, através de uma revisão integrativa de artigos publicados nos anos de 2020 e 2021 em plataformas de dados, como PubMed e ScienceDirect.

\section{Metodologia}

O estudo trata-se de uma pesquisa do tipo revisão integrativa de literatura, de natureza exploratória e descritiva com abordagem qualitativa. Por meio dessa metodologia, é possível analisar os estudos mais relevantes disponíveis na literatura e utilizar as evidencias disponíveis no campo científico de forma sistemática e ordenada, permitindo o aprofundamento do conhecimento do tema realizada por meio de levantamento bibliográfico (Souza; Silva \& Carvalho, 2010). A pesquisa bibliográfica proporciona levantar estudos de referência publicados sobre o tema afim de compilar o conhecimento científico existente, apresentar justificativas para a condução do estudo e identificar lacunas (Conforto; Amaral \& Silva, 2011).

Desse modo, a formulação de uma problemática, que dará início a revisão, é possível ser bem estruturada e delimitada tendo como etapas 1) a enunciação da pergunta de pesquisa; 2) fundamentação teórico-empírica das hipóteses e 3) detalhamento dos modos de investigação - entre eles a apresentação dos critérios de seleção, modo de coleta dos dados e procedimento na análise dos dados (Tunes, 2018).

O levantamento bibliográfico consistiu em acesso a artigos publicados em revistas cientificas eletrônicas da área da saúde, dentre eles a bases de dados PubMed e ScienceDirect tendo como questão norteadora "Qual a relação entre a incidência 
do parto prematuro em gestantes e a COVID-19?”. Para tal, adotou-se a estratégia PICO para o direcionamento da revisão de literatura afim de desenhar o estudo consoante ao tema. Esse acrônimo é representando no presente estudo pelos seguintes itens: P) População (pacientes gestantes com COVID-19); I) Intervenção (parto prematuro); C) Comparação (incidência de partos prematuros antes e após o período de pandemia do COVID-19); O) Desfecho ou resultados (resultado observado com a pesquisa bibliográfica referente ao tema); T) Tempo (estudos publicados nos últimos 2 anos - entre 2020 e 2021) (Rice, 2013).

Os artigos foram selecionados pela leitura criteriosa dos títulos, resumos e, em seguida, dos artigos na íntegra. Os critérios de inclusão definidos foram a seleção de artigos disponibilizados completos, gratuitos, publicados no ano de 2020 e 2021, em língua inglesa, independentemente do método de pesquisa utilizado e que fossem pertinentes ao título estabelecido para este estudo. Foram excluídos da pesquisa, artigos que não foram disponibilizados na integra, publicados antes de 2020, que se encontravam em qualquer outro idioma que não os supracitados, que estavam duplicados e que não se adequavam aos objetivos almejados.

Os termos foram inseridos em inglês, da seguinte forma: "Premature birth" associado pelo operador booleano "AND" aos termos "pregnant women" e "COVID-19". A partir das estratégias de elegibilidade utilizadas foram encontrados 203 artigos, sendo 116 artigos no banco ScienceDirect e 87 no PubMed. Após a leitura dos títulos e dos resumos, foram préselecionados 14 artigos que atendiam aos critérios de inclusão. Em seguida à aplicação dos critérios de exclusão, 3 artigos retirados em razão de duplicidade e/ou não atendimento a proposta desta revisão integrativa. Ao fim, 11 artigos selecionados para comporem o presente trabalho. A Figura 1, exemplifica o número de estudos selecionados conforme a descrição anterior.

Figura 1 - Fluxo da seleção das publicações para análise no presente estudo.

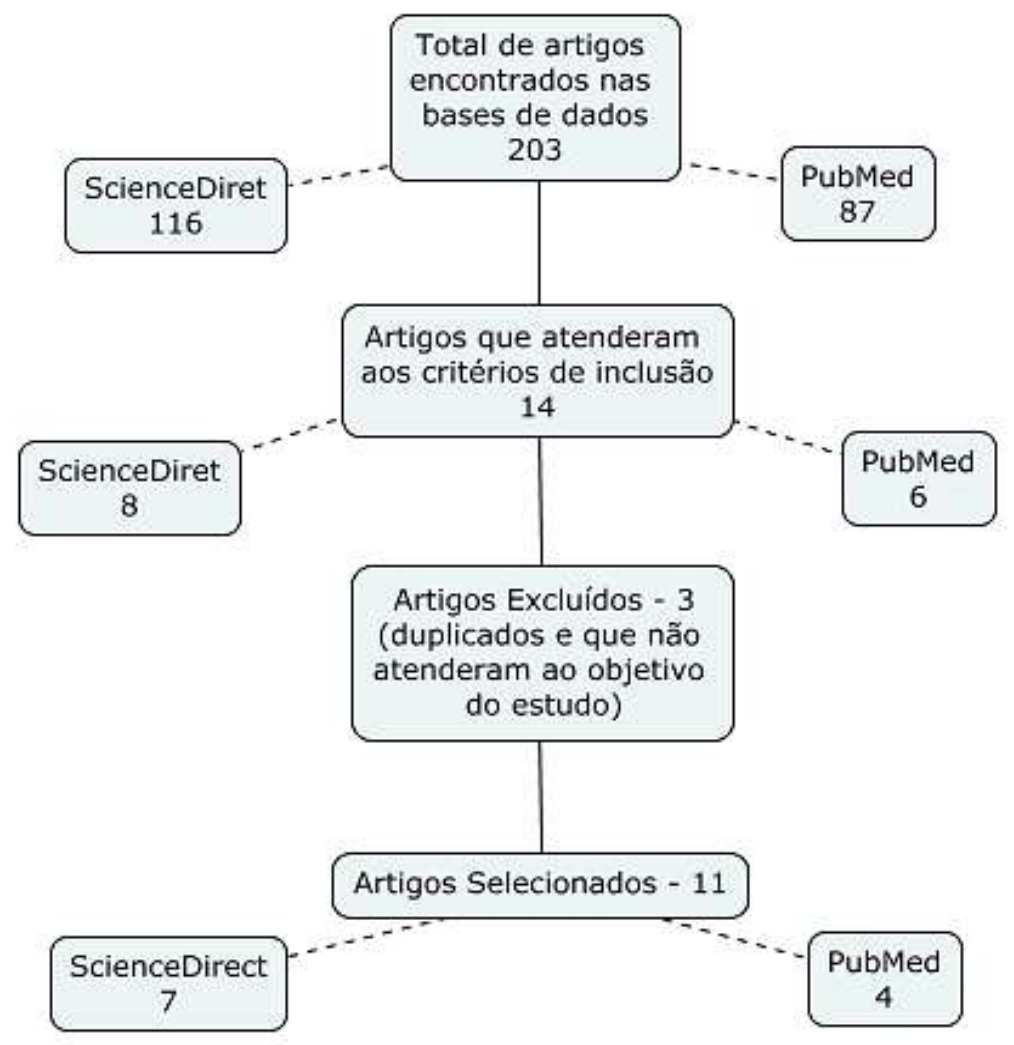

Fonte: Autores. 


\section{Resultados}

O objetivo deste estudo foi apresentar e discutir os achados da literatura referentes à incidência de parto prematuro em gestantes com COVID-19 e a associação da exposição ao vírus e o risco obstétrico. Foi encontrado um total de 203 artigos nas bases de dados, das quais foram utilizadas a plataforma PubMed e ScienceDirect. Excluíram-se aqueles cujas temáticas fugiam do foco do estudo e destes, 14 atenderam ao critério de inclusão e 11 artigos foram elegíveis, sendo 4 artigos da plataforma PubMed e 7 artigos da plataforma ScienceDirect, pois atenderam ao objetivo da pesquisa e constam nas referências da pesquisa e na tabela a seguir. Os artigos analisados foram publicados em periódicos internacionais em 2020 e 2021, todos em língua inglesa. Esses trabalhos selecionados consistem em estudos prospectivos e retrospectivos, relatos de casos, metanálises e revisões sistemáticas. Dessa forma, podemos observar as subcategorias analisadas conforme mostra a Tabela 1, correspondendo aos títulos, autores, ano da publicação, objetivo de estudo e banca de dados.

Tabela 1 - Síntese dos estudos que atenderam aos critérios de elegibilidade, segundo autor e ano de publicação, título, objetivo de estudo e banca de dados.

\begin{tabular}{|c|c|c|c|}
\hline Autores/ Ano & Título & Objetivo de Estudo & Banca de dados \\
\hline $\begin{array}{c}\text { Meaney et al., } \\
2020\end{array}$ & $\begin{array}{l}\text { The impact of COVID-19 on } \\
\text { pregnant women's experiences and } \\
\text { perceptions of antenatal maternity } \\
\text { care, social support, and stress- } \\
\text { reduction strategies. }\end{array}$ & $\begin{array}{c}\text { Analisar através de uma pesquisa a } \\
\text { insatisfação das mulheres grávidas } \\
\text { em relação ao apoio psicológico e } \\
\text { social e sobre os cuidados do pré- } \\
\text { natal. }\end{array}$ & ScienceDirect \\
\hline $\begin{array}{l}\text { Martinez-Perez et } \\
\text { al., } 2021\end{array}$ & $\begin{array}{c}\text { The association between SARS-CoV- } \\
2 \text { infection and preterm delivery: a } \\
\text { prospective study with a } \\
\text { multivariable analysis }\end{array}$ & $\begin{array}{c}\text { Analisar a relação entre a exposição } \\
\text { ao vírus na gravidez e maior risco } \\
\text { obstétrico. }\end{array}$ & PubMed \\
\hline $\begin{array}{c}\text { Easterlin et al., } \\
2020\end{array}$ & $\begin{array}{c}\text { Extremely preterm infant born to a } \\
\text { mother with severe covid-19 } \\
\text { pneumonia }\end{array}$ & $\begin{array}{l}\text { Descrever e analisar o caso de um } \\
\text { bebê prematuro extremo nascido de } \\
\text { uma mãe com pneumonia por } \\
\text { COVID-19. }\end{array}$ & PubMed \\
\hline $\begin{array}{c}\text { Melo \& Araújo, } \\
2020\end{array}$ & $\begin{array}{l}\text { COVID-19 infection in pregnant } \\
\text { women, preterm delivery, } \\
\text { birthweight, and vertical } \\
\text { transmission: a systematic review and } \\
\text { meta- analysis }\end{array}$ & $\begin{array}{l}\text { Realizar uma revisão sistemática } \\
\text { com o objetivo de avaliar a infecção } \\
\text { por COVID-19 em mulheres } \\
\text { grávidas e o risco de nascimento } \\
\text { prematuro e baixo peso ao nascer. }\end{array}$ & PubMed \\
\hline $\begin{array}{c}\text { BLauvelt et al., } \\
2020\end{array}$ & $\begin{array}{l}\text { Acute respiratory distress syndrome } \\
\text { in a preterm pregnant patient with } \\
\text { coronavirus disease } 2019 \text { (COVID- } \\
\text { 19) }\end{array}$ & $\begin{array}{l}\text { Narrar a abordagem de uma } \\
\text { paciente grávida de } 28 \text { semanas } \\
\text { com síndrome do desconforto } \\
\text { respiratório agudo resultante da } \\
\text { infecção por COVID-19. }\end{array}$ & PubMed \\
\hline Been et al., 2020 & $\begin{array}{c}\text { Impact of COVID-19 mitigation } \\
\text { measures on the incidence of preterm } \\
\text { birth: a national quasi-experimental } \\
\text { study }\end{array}$ & $\begin{array}{l}\text { Analisar a redução de partos } \\
\text { prematuros na Holanda e a } \\
\text { implantação de medidas de } \\
\text { contenção da COVID-19 }\end{array}$ & ScienceDirect \\
\hline
\end{tabular}




\begin{tabular}{|c|c|c|c|}
\hline Bloise et al., 2021 & $\begin{array}{l}\text { Expression of severe acute respiratory } \\
\text { syndrome coronavirus } 2 \text { cellentry } \\
\text { genes, angiotensin-converting } \\
\text { enzyme } 2 \text { and transmembrane } \\
\text { protease serine } 2 \text {, in the placenta } \\
\text { across gestation and at the maternal- } \\
\text { fetal interface in pregnancies } \\
\text { complicated by preterm birth or } \\
\text { preeclampsia }\end{array}$ & $\begin{array}{l}\text { Examinar o padrão de entrada de } \\
\text { genes específicos na placenta em } \\
\text { gestações de alto risco, comparando } \\
\text { esse padrão com gestações a termo } \\
\text { de mães infectadas que não tiveram } \\
\text { complicações obstétricas. }\end{array}$ & ScienceDirect \\
\hline $\begin{array}{l}\text { Lemon et al., } \\
2021\end{array}$ & $\begin{array}{l}\text { What is driving the decreased } \\
\text { incidence of preterm birth during the } \\
\text { coronavirus disease } 2019 \text { pandemic? }\end{array}$ & $\begin{array}{c}\text { Investigar possíveis causas para a } \\
\text { diminuição da ocorrência de partos } \\
\text { prematuros durante a pandemia. }\end{array}$ & ScienceDirect \\
\hline $\begin{array}{c}\text { Sentilhes et al., } \\
2020\end{array}$ & $\begin{array}{l}\text { Coronavirus disease } 2019 \text { in } \\
\text { pregnancy was associated with } \\
\text { maternal morbidity and preterm birth }\end{array}$ & $\begin{array}{l}\text { Analisar e relacionar a fisiologia da } \\
\text { gravidez com a maior morbidade } \\
\text { materna e parto prematuro em } \\
\text { mulheres infectadas pela doença. }\end{array}$ & ScienceDirect \\
\hline $\begin{array}{l}\text { Pierce-Williams } \\
\text { et al., } 2020\end{array}$ & $\begin{array}{l}\text { Clinical course of severe and critical } \\
\text { coronavirus disease } 2019 \text { in } \\
\text { hospitalized pregnancies: a United } \\
\text { States cohort study }\end{array}$ & $\begin{array}{c}\text { Descrever por meio de um estudo } \\
\text { de coorte o curso clínico da } \\
\text { infecção grave em mulheres } \\
\text { gestantes com síndrome respiratória } \\
\text { grave. }\end{array}$ & ScienceDirect \\
\hline $\begin{array}{l}\text { Antoun et al., } \\
2020\end{array}$ & $\begin{array}{c}\text { Maternal COVID-19 infection, } \\
\text { clinical characteristics, pregnancy, } \\
\text { and neonatal outcome: A prospective } \\
\text { cohort study }\end{array}$ & $\begin{array}{c}\text { Analisar as consequências da } \\
\text { infecção materna na gravidez e } \\
\text { possíveis complicações neonatais. }\end{array}$ & ScienceDirect \\
\hline
\end{tabular}

Fonte: Autores (2021).

\section{Discussão}

\subsection{Abordagem clínica mediante a realização do pré-natal em pacientes acometidas pela COVID-19}

A princípio, deve-se ressaltar o papel preponderante do pré-natal como mecanismo essencial, quanto à profilaxia e reconhecimento de complicações maternas e fetais, que podem ocorrer no período gestacional e puerpério, de forma a proporcionar uma gravidez sem riscos para a gestante e um desenvolvimento saudável para o feto. Assim, a realização desse procedimento de maneira adequada, tem por objetivo orientar a gestante perante a maternidade, de maneira a fomentar o incentivo ao uso de hábitos saudáveis quanto à nutrição adequada (Ministério da saúde, 2005).

De acordo com o Ministério da saúde (2005), algumas restrições devem estar aliadas, mediante a ingestão de bebidas alcoólicas, tabagismo e consumo de drogas ilícitas bem como a utilização de medicamentos, se necessários, os quais não prejudiquem andamento da gestação. Outrossim, é destacado também, a importância do pré-natal, pois corrobora com o tratamento das manifestações físicas decorrentes da fisiologia da gravidez, além das patologias que a paciente já possua e apresente risco à saúde materno-fetal.

Diante disso, Meaney et al. (2020) descreve durante a realização da pesquisa a necessidade do acompanhamento durante a gestação, principalmente do apoio psicológico e social, considerando que durante esse período a mulher está mais vulnerável a alterações hormonais que influenciam diretamente no humor e possibilitam que a mesma seja acometida pela depressão pós-parto. Durante a análise foi constatado que com o decorrer da pandemia o apoio necessário para as mulheres grávidas estava limitado, sobretudo pelos fatores demográficos, além de não ser permitido o acompanhante durante a realização das consultas de pré-natal. 
À vista disso, é importante salientar, que a realização desse acompanhamento gestacional tem sido cada vez mais cautelosa e fundamental, com o fito de controlar e diminuir complicações advindas do SARS-CoV-2, como o aumento de partos prematuros e mortes. Nessa perspectiva, Nogueira et al. (2020) disserta que as gestantes que apresentam o quadro positivo para COVID-19, urgem um atendimento de qualidade, principalmente na realização do pré-natal, mediante uma análise criteriosa quanto ao histórico clínico individual de cada paciente. Assim, a consulta para o monitoramento da saúde da gestante e do feto deve ser feita de acordo com a idade gestacional e com a gravidade da doença, a mesma deve ser orientada a fazer o isolamento e o tratamento sugerido pela obstetra (Bherimg et al., 2021).

Em virtude do SARS-CoV-2 ser uma patologia que surgiu há pouco tempo, alguns estudos entraram em divergências de pensamento, sobre a incidência de partos prematuros e danos à saúde da gestante e do feto. Segundo Melo (2020), as pacientes grávidas quando em contato com o vírus, podem sim ter um aumento em relação ao risco do parto prematuro, devido aos danos fisiológicos na saúde da gestante. Entretanto, Lemonet al. (2021), descreve que houve uma diminuição na taxa de partos prematuros em 2020 em Pittsburgh, provavelmente pelo maior acompanhamento do pré-natal, visto que houve uma menor incidência em mulheres que viviam em lugares mais favorecidos.

Todavia, apesar do Lemonet al. (2021) relaciona a queda dos partos prematuros ao alto status socioeconômico, Beenet al. (2020) através de uma análise desenvolvida na Holanda, confirmou que houve sim redução em locais com maior acesso a saúde de qualidade, porém não foi estatisticamente significativo. Percebe-se que durante os resultados da pesquisa as medidas de mitigação da COVID-19 juntamente com o aumento da preocupação em relação aos cuidados com os pré-natais, foram extremamente importantes para a diminuição de partos prematuros.

Segundo Donderset al. (2020), quando relacionado a patologia com a gestação, pode haver um comprometimento no estado fisiológico da gestante que pode resultar em prejuízos ao feto, tornando toda mulher grávida um paciente de alto risco para a COVID-19. Nesse cenário, devido aos efeitos causados pelo SARS-CoV-2, as pacientes podem estar mais propensas a manifestar um quadro de pré-eclâmpsia, e consequentemente, a viabilidade de um parto prematuro ou óbito. Nesse contexto, é importante destacar que os pacientes infectados possuem potencial para desenvolver uma resposta inflamatória exacerbada, o que resulta num estresse oxidativo e uma tempestade de citocina (Dana et al., 2020).

Ademais, nos casos de pacientes positivadas para o vírus em questão, há a necessidade do seguimento de protocolos de isolamento domiciliar, contudo, que seja feito a notificação da infecção para que os responsáveis pelo atendimento possam utilizar de mecanismos que garantam o monitoramento e acompanhamento da gestação. Para tanto, essa gestante deve ser monitorada desde sua frequência respiratória, sintomas gerais, saturação de oxigênio, pressão arterial como medida profilática para uma detecção precoce de quaisquer alterações. Cabe ainda pontuar, que caso haja a necessidade, uma equipe de saúde munida de todos os EPI's, necessários para a realização dos procedimentos, realize o atendimento domiciliar, de maneira segura, tanto para os profissionais, quanto para a paciente (Brasil, 2020).

\subsection{Possíveis complicações geradas pela síndrome respiratória aguda por SARS-CoV-2 para a gestante e o recém- nascido prematuro}

Mediante uma análise crítica dos pesquisadores de distintos países e em diferentes períodos da pandemia, nota-se um estudo inicial acerca da temática proporcionado por um espaço amostral reduzido. Portanto, essa perspectiva não necessariamente se mostra de maneira fidedigna com a realidade social. Duas linhas de estudos se desenvolvem perante o tema contrapondo-se. Enquanto algumas pesquisas como as de Martinez-Perez et al. (2021), Rodriguez et al. (2021) e Sentilhes et al. (2020) mostram uma associação entre a infecção por Coronavírus com à morbidade materna e parto prematuro. Outros estudos, como o de Melo et al. (2020) relatam que o COVID-19 em mulheres grávidas pode não estar associado à ocorrência de partos prematuros. 
Diante o exposto, acredita-se que as alterações fisiológicas resultantes da gestação que proporcionam possíveis complicações na sintomatologia do COVID-19, podendo acarretar na gestante um quadro de eclâmpsia, partos prematuros ou urgência de um parto cesáreo (Martinez-Perez et al., 2021). Contudo, estudos levam a compreensão da inexistência de maneira assertiva no que diz respeito à relação do COVID-19 com as complicações gestacionais e neonatais (Melo, 2020).

A relação do SARS-CoV-2 com o parto prematuro, observou-se que, a interação do organismo da mulher com o vírus pode promover sinais e sintomas graves, como problemas pulmonares, que caso se agravarem podem levar a óbito. Além disso, pode contribuir de forma significativa para o agravo da saúde da gestante e do feto (Sentilhes et al., 2020). Somado a isso, Pierce-Williams et al. (2020), afirma que, caso gestantes contraiam o vírus, podem acarretar em abortos espontâneos, sofrimento fetal e parto prematuros, incluído aos outros fatores maternos sistêmicos desencadeados pela doença.

De acordo com um estudo de Coorte feito nos EUA, foi constatado que o parto prematuro acontecia devido às complicações causadas pela doença como: dispneia, frequência respiratória aumentada, saturação menor que $93 \%$ e em casos mais graves, insuficiência respiratória. Como consequência, houve um aumento considerável no número de cesarianas realizadas em decorrência do contato do vírus com a gestante. Ademais, o fato de a puérpera apresentar alguma comorbidade como doença pulmonar, doença cardíaca e obesidade, contribuiu para o agravo e acelerar todas as consequências apresentadas anteriormente (Pierce-Williams et al., 2020).

Outro ponto observado nas pesquisas de Blauvelt et al. (2020) foi a dificuldade no tratamento da Síndrome do desconforto respiratório agudo (SDRA) por infecção por COVID-19 em grávidas, devido às mudanças fisiológicas normais da gravidez, como a ventilação minuto aumentada, o deslocamento do diafragma, a complacência da parede torácica e o consumo de oxigênio aumentado, e essa dificuldade no tratamento, pode acarretar um parto pré-termo, mas de acordo com esse estudo, as puérperas não apresentam riscos maiores de desenvolver doença grave em comparação com pacientes não grávidas.

Portanto, por ser uma patologia nova ainda com poucas evidências científicas não foi chegado a um consenso sobre a correlação da infecção por Coronavírus e o parto prematuro em gestantes, mas podemos por meio do estudo realizado, chegar conclusão que há alterações significativas em mulheres infectadas que manifestam a doença em grau moderado ou severo, entretanto não há evidências de alterações obstétricas em infecções leves.

\section{Conclusão}

Por meio dos estudos analisados percebe-se que a ocorrência entre COVID-19 e gestação pode estar relacionado ao maior risco de complicações, tendo como efeito um parto cada vez mais precoce, possibilitando um parto prematuro, devido uma resposta inflamatória exacerbada, insuficiência respiratória, quadro de pré-eclâmpsia ou sofrimento fetal. Além disso, é evidenciado que apesar da dificuldade em tratar as gestantes com síndrome do desconforto respiratório agudo por infecção COVID-19 devido às mudanças fisiológicas no organismo, essas não apresentam maior risco de evoluírem para gravidade quando comparadas às pacientes não grávidas.

Apesar de certas análises demonstrarem redução dos casos de parto prematuro, é percebido o maior acompanhamento gestacional na localidade e assim o tratamento precoce e reversão do quadro que gere risco à saúde materno-fetal. Além do que houve mudanças nas medidas comportamentais e higiênicas da população socioeconômica estudada prevenindo o risco de infecção pelo vírus COVID-19.

Durante a realização da revisão e síntese dos principais achados no banco científico em busca de discutir os dados referentes à incidência de parto prematuro em gestantes com COVID-19 e a associação da exposição ao vírus e o risco obstétrico, destaca-se como limitação a incipiência de artigos científicos que contemplem essa temática. Até o momento as evidências são conflitantes e incertas, sendo percebida a falta de integração de dados comparáveis entre os países. 
Por essa razão, recomenda-se a realização de pesquisas futuras sobre a referida temática na perspectiva de suprir lacunas importantes nessa área, sendo necessário mais estudos para compreender o impacto do vírus na gravidez e nos resultados neonatais afim de guiar a política pública de saúde para gestantes e recém-nascidos no atendimento clínico, além de fundamentar tomadas de decisões que visem o bem-estar e assistência a essa classe da sociedade diante o novo cenário surgido com o vírus COVID-19.

\section{Referências}

Allotey, J., Stallings, E., Bonet, M., Yap, M., Chatterjee, S., Kew, T., ... \& PregCOV-19 Living Systematic Review Consortium. (2020). Clinical manifestations, risk factors, and maternal and perinatal outcomes of coronavirus disease 2019 in pregnancy: living systematic review and metaanalysis. $B m j, 370$.

Antoun, L., El Taweel, N., Ahmed, I., Patni, S., \& Honest, H. (2020). Maternal COVID-19 infection, clinical characteristics, pregnancy, and neonatal outcome: A prospective cohort study. European Journal of Obstetrics \& Gynecology and Reproductive Biology, 252, 559-562.

Bhering, N. B. V., Arndt, C. G., de Paiva Gonçalves Filho, D. A., Vita, D. T. P., da Cunha Chagas, F. R., Gazzoni, G. A. S., ... \& da Costa, T. M. M. (2021). Covid-19-induced preterm birth: a literature review. Brazilian Journal of Health Review, 4(2), 4401-4415.

Been, J. V., Ochoa, L. B., Bertens, L. C., Schoenmakers, S., Steegers, E. A., \& Reiss, I. K. (2020). Impact of COVID-19 mitigation measures on the incidence of preterm birth: a national quasi-experimental study. The Lancet Public Health, 5(11), e604-e611.

Blauvelt, C. A., Chiu, C., Donovan, A. L., Prahl, M., Shimotake, T. K., George, R. B., ... \& Gaw, S. L. (2020). Acute respiratory distress syndrome in a preterm pregnant patient with coronavirus disease 2019 (COVID-19). Obstetrics \& Gynecology, 136(1), 46-51.

Bloise, E., Zhang, J., Nakpu, J., Hamada, H., Dunk, C. E., Li, S., ... \& Lye, S. J. (2021). Expression of severe acute respiratory syndrome coronavirus 2 cell entry genes, angiotensin-converting enzyme 2 and transmembrane protease serine 2 , in the placenta across gestation and at the maternal-fetal interface in pregnancies complicated by preterm birth or preeclampsia. American journal of obstetrics and gynecology, 224(3), 298-e1.

Conforto, E. C., Amaral, D. C., \& Silva, S. D. (2011). Roteiro para revisão bibliográfica sistemática: aplicação no desenvolvimento de produtos e gerenciamento de projetos. Trabalho apresentado, 8.

Costa, R. E. A. R., Pompeu, J. G. F., Querido, Á. C. C. M., Campos, L. N. R., Calaça, M. B., da Silva, A. N., ... \& dos Santos Sousa, F. W. (2020). Principais Complicações Relacionadas à COVID-19 na Gravidez. Research, Society and Development, 9(8), e490985880-e490985880.

Silva, C. C., de Carvalho, C. M. O., de Lima, D. C., Costa, E. S., de Andrade, V. M. B., Tenorio, B. M., ... \& Tenorio, F. C. A. M. (2021). Covid-19: Aspectos da origem, fisiopatologia, imunologia e tratamento-uma revisão narrativa. Revista Eletrônica Acervo Saúde, 13(3), e6542-e6542.

Donders, F., Lonnée-Hoffmann, R., Tsiakalos, A., Mendling, W., Martinez de Oliveira, J., Judlin, P., ... \& Workgroup, G. (2020). ISIDOG recommendations concerning COVID-19 and pregnancy. Diagnostics, 10(4), 243.

Easterlin, M. C., De Beritto, T., Yeh, A. M., Wertheimer, F. B., \& Ramanathan, R. (2020). Extremely preterm infant born to a mother with severe COVID-19 pneumonia. Journal of Investigative Medicine High Impact Case Reports, 8, 2324709620946621.

ESTRELA, F., Silva, K. K. A. D., Cruz, M. A. D., \& Gomes, N. P. (2020). Gestantes no contexto da pandemia da Covid-19: reflexões e desafios. Physis: Revista de Saúde Coletiva, 30, e300215.

Lemon, L., Edwards, R. P., \& Simhan, H. N. (2021). What is driving the decreased incidence of preterm birth during the coronavirus disease 2019 pandemic?. American Journal of Obstetrics \& Gynecology MFM, 3(3), 100330.

Martinez-Perez, O., Rodriguez, P. P., Hernandez, M. M., Pardilla, M. B. E., Perez, N. P., Hernandez, M. R. V., ... \& Bueno, J. A. S. (2021). The association between SARS-CoV-2 infection and preterm delivery: a prospective study with a multivariable analysis. BMC pregnancy and childbirth, 21(1), 1-11.

Mascarenhas, V. H. A., Caroci-Becker, A., Venâncio, K. C. M. P., Baraldi, N. G., Durkin, A. C., \& Riesco, M. L. G. (2020). COVID-19 e a produção de conhecimento sobre as recomendações na gravidez: revisão de escopo. Revista Latino-Americana de Enfermagem, 28.

Meaney, S., Leitao, S., Olander, E. K., Pope, J., \& Matvienko-Sikar, K. (2021). The impact of COVID-19 on pregnant womens' experiences and perceptions of antenatal maternity care, social support, and stress-reduction strategies. Women and Birth.

Melo, G. C. D., \& Araújo, K. C. G. M. D. (2020). COVID-19 infection in pregnant women, preterm delivery, birth weight, and vertical transmission: a systematic review and meta-analysis. Cadernos de saude publica, 36, e00087320.

de Santiago Nogueira, C. M. C., de Alcantara, J. R., Goes, H. M., Costa, S., Morais, F. R. R., Bezerra, K. P., \& de Melo Fialho, A. V. (2020). Análise nacional do perfil das gestantes acometidas pela COVID-19. Brazilian Journal of Health Review, 3(5), 14267-14278.

Ministério da saúde. Departamento de ações programáticas estratégicas. Atenção às Gestantes no Contexto da Infecção COVID 19 causada pelo Novo Coronavírus (SARS-CoV-2), Brasília, 08 de abril. 2020. Disponível em: https://saude.mg.gov.br/images/noticias_e_eventos/000_2020/mar_abr_maio/1404_NOTA-TECNICA-N-72020_COSMU_08_04.pdf. Acesso em: 17 de junho de 2021.

Pierce-Williams, R. A., Burd, J., Felder, L., Khoury, R., Bernstein, P. S., Avila, K., ... \& Berghella, V. (2020). Clinical course of severe and critical coronavirus disease 2019 in hospitalized pregnancies: a United States cohort study. American journal of obstetrics \& gynecology MFM,2(3), 100134. 
Research, Society and Development, v. 10, n. 12, e517101220762, 2021 (CC BY 4.0) | ISSN 2525-3409 | DOI: http://dx.doi.org/10.33448/rsd-v10i12.20762

Pirjani, R., Hosseini, R., Soori, T., Rabiei, M., Hosseini, L., Abiri, A., ... \& Sepidarkish, M. (2020). Maternal and neonatal outcomes in COVID-19 infected pregnancies: a prospective cohort study. Journal of travel medicine, 27(7), taaa158.

Rice, M. J. (2013). Evidence-based practice: a model for clinical application. Journal of the American Psychiatric Nurses Association, 19(4), $217-221$.

Sentilhes, L., De Marcillac, F., Jouffrieau, C., Kuhn, P., Thuet, V., Hansmann, Y., ... \& Deruelle, P. (2020). Coronavirus disease 2019 in pregnancy was associated with maternal morbidity and preterm birth. American journal of obstetrics and gynecology, 223(6), 914-e1.

Silva, A. W. C., Cunha, A. A., Alves, G. C., Corona, R. A., de Mattos Dias, C. A. G., Nassiri, R., ... \& Fecury, A. A. (2020). Caracterização clínica e epidemiologia de 1560 casos de COVID-19 em Macapá/AP, extremo norte do Brasil. Research, Society and Development, $9(8)$, e150985499-e150985499.

Sousa, G. O., Sales, B. N., Rodrigues, A. M. X., de Moura Rocha, G. M., \& de Oliveira, G. A. L. (2020). Evolução epidemiológica da COVID-19 no Brasil e no mundo. Research, Society and Development, 9(7), e630974653-e630974653.

Souza, M. T. D., Silva, M. D. D., \& Carvalho, R. D. (2010). Integrative review: what is it? How to do it?. Einstein (São Paulo), 8, $102-106$.

Su, S., Wong, G., Shi, W., Liu, J., Lai, A. C., Zhou, J., ... \& Gao, G. F. (2016). Epidemiology, genetic recombination, and pathogenesis of coronaviruses. Trends in microbiology, 24(6), 490-502.

Water, W. H. O. (2020). sanitation, hygiene, and waste management for the COVID-19 virus. Published online March.

Trapani Júnior, A., Vanhoni, L. R., Marcolin, A. C., \& Silveira, S. K. (2020). Protocolo de atendimento no parto, puerpério e abortamento durante a pandemia da COVID-19. Femina.

Tunes, E. Formulação do problema de investigação científica e elaboração do projeto de pesquisa: orientações breves para o estudante.

Vieira, J. M., Ricardo, O. M. D. P., Hannas, C. M., Kanadani, T. C. M., Prata, T. D. S., \& Kanadani, F. N. (2020). What do we know about COVID-19? A review article. Revista da Associação Médica Brasileira, 66, 534-540.

Zaigham, M., \& Andersson, O. (2020). Maternal and perinatal outcomes with COVID-19: a systematic review of 108 pregnancies. Acta obstetricia et gynecologica Scandinavica, 99(7), 823-829. 